PROCEEDINGS OF THE

AMERICAN MATHEMATICAL SOCIETY

Volume 139, Number 4, April 2011, Pages 1483-1494

S 0002-9939(2010)10568-3

Article electronically published on September 2, 2010

\title{
EXPONENTIAL SCALE MIXTURE OF MATRIX VARIATE CAUCHY DISTRIBUTION
}

\author{
AMADOU SARR AND ARJUN K. GUPTA
}

(Communicated by Edward C. Waymire)

\begin{abstract}
In this paper, we introduce a new subclass of matrix variate elliptically contoured distributions that are obtained as a scale mixture of matrix variate Cauchy distribution and exponential distribution. We investigate its properties, such as stochastic representation and characteristic function. Unlike Cauchy distribution, it is shown that the generating variate of the new distribution possesses finite moments. The distributions of the unbiased estimators of $\boldsymbol{\mu}$ and $\boldsymbol{\Sigma}$ are derived. Furthermore, an identity involving a special function with a matrix argument is also obtained.
\end{abstract}

\section{INTRODUCTION}

In recent years, much attention has been paid to elliptically contoured distributions. The most important results related to vector variate elliptical distributions can be found in the book by Fang et al. (1990), while matrix variate elliptical distributions have been studied by Fang and Zhang (1990), and Gupta and Varga (1993), among others. Fang and Anderson (1990) include a large number of references before 1990 in this area. The following definition is taken from Gupta and Varga (1993).

Definition 1.1. Let $\mathbf{X}$ be a $p \times n$ random matrix. Then $\mathbf{X}$ is said to have a matrix variate elliptically contoured (m.e.c.) distribution if its characteristic function has the form

(1)

$$
\begin{array}{r}
\phi_{\mathbf{X}}(\mathbf{T})=\operatorname{etr}\left(i \mathbf{T}^{\prime} \mathbf{M}\right) \psi\left[\operatorname{tr}\left(\mathbf{T}^{\prime} \mathbf{\Sigma} \mathbf{T} \mathbf{\Phi}\right)\right] \text { with } \mathbf{T} \in \mathcal{R}^{p \times n}, \mathbf{M} \in \mathcal{R}^{p \times n}, \boldsymbol{\Sigma} \in \mathcal{R}^{p \times p}, \\
\mathbf{\Phi} \in \mathcal{R}^{n \times n}, \mathbf{\Sigma} \geq \mathbf{O}, \mathbf{\Phi} \geq \mathbf{O} \text { and } \psi:[0, \infty[\rightarrow \mathbb{R} .
\end{array}
$$

The matrices $\mathbf{M}, \boldsymbol{\Sigma}$ and $\boldsymbol{\Phi}$ are the parameters of the distribution, and $\operatorname{etr}(\cdot)$ denotes the exponential of the trace function.

This distribution is denoted by $\mathbf{X} \sim E_{p, n}(\mathbf{M}, \boldsymbol{\Sigma} \otimes \mathbf{\Phi}, \psi)$. The function $\psi$ is called the characteristic generator (c.g.). As a special case, when $\psi(\cdot)$ is specified by $\psi(z)=\exp \left(-\frac{z}{2}\right)$, then $\mathbf{X}$ has a matrix variate normal distribution. The class of elliptically contoured distributions includes the familiar normal one and shares

Received by the editors December 14, 2009 and, in revised form, May 1, 2010.

2010 Mathematics Subject Classification. Primary 62H10; Secondary 62H12.

Key words and phrases. Matrix variate Cauchy, scale mixture distribution, exponential distribution, stochastic representation, Whittaker's function, Meijer's function.

(C)2010 American Mathematical Society 
many of its properties. In particular, this class possesses the linearity property. In other words, any linear combination of elliptical distributions is another elliptical distribution with the same (c.g.) $\psi$. If $n=1$, then $\mathbf{x} \sim E_{p}(\mathbf{m}, \boldsymbol{\Sigma}, \psi)$ is said to have a vector variate elliptical distribution. The relationship, in terms of the distributions, of the matrix and the vector is illustrated as follows:

(2)

$\mathbf{X} \sim E_{p, n}(\mathbf{M}, \boldsymbol{\Sigma} \otimes \mathbf{\Phi}, \psi)$ if and only if $\mathbf{x}=\operatorname{vec}\left(\mathbf{X}^{\prime}\right) \sim E_{p n}\left(\operatorname{vec}\left(\mathbf{M}^{\prime}\right), \boldsymbol{\Sigma} \otimes \mathbf{\Phi}, \psi\right)$.

Here, $\operatorname{vec}(\mathbf{A})$ is defined by

$$
\operatorname{vec}(\mathbf{A})=\left(\begin{array}{c}
\mathbf{a}_{1} \\
\mathbf{a}_{2} \\
\vdots \\
\mathbf{a}_{n}
\end{array}\right),
$$

where $\mathbf{a}_{1}, \ldots, \mathbf{a}_{n}$ denote the columns of the $p \times n$ matrix $\mathbf{A}$. Following Schoenberg (1938), Fang and Anderson (1982) derived a stochastic representation of matrix variate elliptical distributions as follows.

Theorem 1.1. Let $\mathbf{X}$ be a $p \times n$ random matrix. Let $\mathbf{M}$ be $p \times n, \boldsymbol{\Sigma}$ be $p \times p$ and $\boldsymbol{\Phi}$ be $n \times n$ constant matrices, with $\boldsymbol{\Sigma} \geq \mathbf{O}, \boldsymbol{\Phi} \geq \mathbf{O}, \operatorname{rank}(\boldsymbol{\Sigma})=p_{1}$, and $\operatorname{rank}(\boldsymbol{\Phi})=n_{1}$. Then

$$
\mathbf{X} \sim E_{p, n}(\mathbf{M}, \boldsymbol{\Sigma} \otimes \mathbf{\Phi}, \psi)
$$

if and only if

$$
\mathbf{X} \stackrel{d}{=} \mathbf{M}+R \mathbf{A} \mathbf{U} \mathbf{B}^{\prime}
$$

where $\mathbf{U}$ is $p_{1} \times n_{1}$ and $\operatorname{vec}\left(\mathbf{U}^{\prime}\right)$ is uniformly distributed on the unit sphere $S_{p_{1} n_{1}}$, $R$ is a nonnegative random variable, $R$ and $\mathbf{U}$ are independent, $\boldsymbol{\Sigma}=\mathbf{A} \mathbf{A}^{\prime}$ and $\mathbf{\Phi}=\mathbf{B B}^{\prime}$ are rank factorizations of $\boldsymbol{\Sigma}$ and $\mathbf{\Phi}$. Moreover

$$
\psi(y)=\int_{0}^{\infty} \Omega_{p_{1} n_{1}}\left(R^{2} y\right) d F(R), y \geq 0,
$$

where $\Omega_{p_{1} n_{1}}\left(\mathbf{t}^{\prime} \mathbf{t}\right), \mathbf{t} \in \mathbb{R}^{p_{1} n_{1}}$, denotes the characteristic generator of $\operatorname{vec}\left(\mathbf{U}^{\prime}\right)$ and $F(r)$ denotes the distribution function of $R$.

The expression $\mathbf{M}+R \mathbf{A} \mathbf{U B}^{\prime}$ is called the stochastic representation of $\mathbf{X}$. In relation (3), the notation $\stackrel{d}{=}$ stands for "equality in distribution". The random matrix $\mathbf{X}$ does not, in general, possess a probability density function (p.d.f.). But if it does, it will have the following form (see Gupta and Varga (1993), p. 26):

$$
f(\mathbf{X})=|\boldsymbol{\Sigma}|^{-\frac{n}{2}}|\mathbf{\Phi}|^{-\frac{p}{2}} h\left(\operatorname{tr}\left((\mathbf{X}-\mathbf{M})^{\prime} \boldsymbol{\Sigma}^{-\mathbf{1}}(\mathbf{X}-\mathbf{M}) \boldsymbol{\Phi}^{-\mathbf{1}}\right)\right),
$$

where $\boldsymbol{\Sigma}>\mathbf{O}$ and $\boldsymbol{\Phi}>\mathbf{O}$. The function $h$ is called the density generator of the distribution. For example, we obtain the matrix variate Pearson type VII distribution if we take

$$
h(z)=\frac{\Gamma(q)}{(m \pi)^{\frac{n p}{2}} \Gamma\left(q-\frac{n p}{2}\right)}\left(1+\frac{z}{m}\right)^{-q}
$$

with $m>0$ and $q>\frac{n p}{2}$. This subclass of matrix variate elliptical distributions includes the well known matrix variate $t$-distributions, and therefore Cauchy distributions as special cases. Throughout this paper, we focus our attention on the matrix variate elliptical distribution that possesses a density. The main aim of this 
work is to introduce and investigate a subclass of matrix variate elliptical distributions that is obtained from Cauchy distribution by using a method of mixture. Indeed, such a method allows one to generate other subclasses of matrix variate distributions. For the vector case, this is defined in the book by Muirhead (1982). Gupta and Varga (1993) gave an extension of the above-quoted result for the matrix variate elliptical distributions.

The paper is organized as follows: In section 2, we will define the subclass of the scale mixture of Cauchy distribution (SMC) and provide its density. Some properties of SMC distributions will be investigated in section 3. Finally the distributions of the unbiased estimators of $\boldsymbol{\mu}$ and the scale matrix $\boldsymbol{\Sigma}$ are obtained in section 4 .

\section{Scale miXture-CAuchy Distribution}

Before giving the definition of this distribution, we need the following result, due to Gupta and Varga (1993).

Theorem 2.1. Let $\mathbf{X} \sim E_{p, n}(\mathbf{M}, \boldsymbol{\Sigma} \otimes \mathbf{\Phi}, \psi)$ (with $\left.\boldsymbol{\Sigma}, \boldsymbol{\Phi}>\mathbf{O}\right)$ have the p.d.f.

$$
f(\mathbf{X})=\frac{1}{|\boldsymbol{\Sigma}|^{\frac{n}{2}}|\mathbf{\Phi}|^{\frac{p}{2}}} h\left(\operatorname{tr}\left((\mathbf{X}-\mathbf{M})^{\prime} \boldsymbol{\Sigma}^{-1}(\mathbf{X}-\mathbf{M}) \mathbf{\Phi}^{-1}\right)\right) .
$$

Suppose $G(z)$ is a distribution function on $(0, \infty)$. Let

$$
g(\mathbf{X})=\frac{1}{|\boldsymbol{\Sigma}|^{\frac{n}{2}}|\mathbf{\Phi}|^{\frac{p}{2}}} \cdot \int_{0}^{\infty} z^{-\frac{p n}{2}} h\left(\frac{1}{z} \operatorname{tr}\left((\mathbf{X}-\mathbf{M})^{\prime} \boldsymbol{\Sigma}^{-1}(\mathbf{X}-\mathbf{M}) \mathbf{\Phi}^{-1}\right)\right) d G(z) .
$$

Then, $g(\mathbf{X})$ is also the p.d.f. of an m.e.c. distribution.

The proof can be found in Gupta and Varga (1993), pp. 78-79. As a special case, when the density generator $h(\cdot)$ in (6) is specified by

$$
h(y)=(2 \pi)^{-\frac{n p}{2}} \exp \left(-\frac{y}{2}\right)
$$

then the distribution of $\mathbf{X}$ is said to be a scale mixture of normal distribution. Note that many elliptical distributions belong to the family of scale mixture of normal distribution. For the vector case, a detailed study of normal mixture distribution can be found in the book by Muirhead (1982).

Definition 2.1. The $p \times n$ random matrix $\mathbf{X}$ is said to have a matrix variate Cauchy (m.v.c.) distribution with parameters $\mathbf{M}: p \times n, \boldsymbol{\Sigma}: p \times p, \boldsymbol{\Phi}: n \times n$ with $\boldsymbol{\Sigma}>\mathbf{O}$, and $\boldsymbol{\Phi}>\mathbf{O}$ if its p.d.f. is

$$
f(\mathbf{X})=\frac{\Gamma\left(\frac{n p+1}{2}\right)}{\pi^{\frac{n p+1}{2}}|\boldsymbol{\Sigma}|^{\frac{n}{2}}|\mathbf{\Phi}|^{\frac{p}{2}}}\left(1+\operatorname{tr}\left[(\mathbf{X}-\mathbf{M})^{\prime} \boldsymbol{\Sigma}^{-1}(\mathbf{X}-\mathbf{M}) \mathbf{\Phi}^{-1}\right]\right)^{-\frac{n p+1}{2}} .
$$

Its density generator $h(\cdot)$ is given by

$$
h(y)=c_{n p}(1+y)^{-\frac{n p+1}{2}}, \text { where } c_{n p}=\frac{\Gamma\left(\frac{n p+1}{2}\right)}{\pi^{\frac{n p+1}{2}}} .
$$

Remark 2.1. The matrix variate Cauchy distribution can be expressed as a scale mixture of matrix variate normal distribution. Indeed, consider the Lévy distribution function $G(z)$, with parameters 1 and 0 . That is,

$$
d G(z)=\frac{1}{\sqrt{2 \pi}} \frac{1}{z^{3 / 2}} \exp \left(-\frac{1}{2 z}\right) d z, z>0 .
$$


Hence, the corresponding density generator $\tilde{h}(\cdot)$ of scale mixture of normal distribution is given by

$$
\begin{aligned}
\tilde{h}(u) & =\frac{1}{(2 \pi)^{\frac{n p}{2}}} \int_{0}^{\infty} z^{-\frac{n p}{2}} \exp \left(-\frac{u}{2 z}\right) \cdot \frac{1}{\sqrt{2 \pi}} \frac{1}{z^{3 / 2}} \exp \left(-\frac{1}{2 z}\right) d z \\
& =\frac{1}{(2 \pi)^{\frac{n p+1}{2}}} \int_{0}^{\infty} z^{-\frac{n p+3}{2}} \exp \left(-\frac{1+u}{2 z}\right) d z
\end{aligned}
$$

By making the change of variable $y=\frac{1+u}{2 z}$, we get

$$
\begin{aligned}
\int_{0}^{\infty} z^{-\frac{n p+3}{2}} \exp \left(-\frac{1+u}{2 z}\right) d z & =-\int_{\infty}^{0}\left(\frac{1+u}{2 y}\right)^{-\frac{n p+3}{2}} \exp (-y) \frac{1+u}{2} \frac{d y}{y^{2}} \\
& =\int_{0}^{\infty}\left(\frac{1+u}{2}\right)^{-\frac{n p+1}{2}} y^{\frac{n p+1}{2}-1} \exp (-y) d y \\
& =\left(\frac{1+u}{2}\right)^{-\frac{n p+1}{2}} \underbrace{\int_{0}^{\infty} y^{\frac{n p+1}{2}-1} \exp (-y) d y}_{=\Gamma\left(\frac{n p+1}{2}\right)}
\end{aligned}
$$

Consequently, we have

$$
\begin{aligned}
\tilde{h}(u) & =\frac{1}{(2 \pi)^{\frac{n p+1}{2}}} 2^{\frac{n p+1}{2}}(1+u)^{-\frac{n p+1}{2}} \Gamma\left(\frac{n p+1}{2}\right) \\
& =\frac{\Gamma\left(\frac{n p+1}{2}\right)}{\pi^{\frac{n p+1}{2}}}(1+u)^{-\frac{n p+1}{2}} .
\end{aligned}
$$

We now define the scale mixture of matrix variate Cauchy distribution.

Definition 2.2. The $p \times n$ random matrix $\mathbf{X}$ is said to have an exponential scale mixture of matrix variate Cauchy distribution with parameters $\mathbf{M}: p \times n$, $\boldsymbol{\Sigma}: p \times p, \boldsymbol{\Phi}: n \times n$ with $\boldsymbol{\Sigma}>\mathbf{O}$, and $\boldsymbol{\Phi}>\mathbf{O}$ if its p.d.f. is

$$
g(\mathbf{X})=\frac{1}{|\boldsymbol{\Sigma}|^{\frac{n}{2}}|\mathbf{\Phi}|^{\frac{p}{2}}} \cdot \int_{0}^{\infty} z^{-\frac{p n}{2}} h\left(\frac{1}{z} \operatorname{tr}\left((\mathbf{X}-\mathbf{M})^{\prime} \boldsymbol{\Sigma}^{-1}(\mathbf{X}-\mathbf{M}) \Phi^{-1}\right)\right) d G(z),
$$

where $h(\cdot)$ is specified by (9), and $d G(z)=\lambda \exp (-\lambda z) d z, \lambda>0$. We shall denote it by $\mathbf{X} \sim S M C_{p, n}(\mathbf{M}, \boldsymbol{\Sigma} \otimes \mathbf{\Phi}, \psi)$.

Our objective is to find the closed-form density generator, denoted by $h_{1}(\cdot)$, for the new subclass obtained by mixing the matrix variate Cauchy with exponential distribution. Here, $h_{1}(\cdot)$ is defined by

$$
h_{1}(u)=\int_{0}^{\infty} z^{-\frac{n p}{2}} h\left(\frac{u}{z}\right) d G(z)
$$


where $h(\cdot)$ is specified by (9), and $d G(z)=\lambda \exp (-\lambda z) d z, \lambda>0, z \in(o, \infty)$. So

$$
\begin{aligned}
h_{1}(u) & =\lambda c_{n p} \int_{0}^{\infty} z^{-\frac{n p}{2}}\left(1+\frac{u}{z}\right)^{-\frac{n p+1}{2}} e^{-\lambda z} d z \\
& =\lambda c_{n p} \int_{0}^{\infty} z^{-\frac{n p}{2}} z^{\frac{n p+1}{2}}(z+u)^{-\frac{n p+1}{2}} e^{-\lambda z} d z \\
& =\lambda c_{n p} \int_{0}^{\infty} z^{\frac{1}{2}}(z+u)^{-\frac{n p+1}{2}} e^{-\lambda z} d z
\end{aligned}
$$

The explicit expression of the density generator of the new subclass of elliptical distributions is given in the theorem below.

Theorem 2.2. Let $\mathbf{X}$ be a $p \times n$ random matrix. Assume that the p.d.f. of $\mathbf{X}$ is

$$
g(\mathbf{X})=\frac{1}{|\boldsymbol{\Sigma}|^{\frac{n}{2}}|\boldsymbol{\Phi}|^{\frac{p}{2}}} \cdot \int_{0}^{\infty} z^{-\frac{p n}{2}} h\left(\frac{1}{z} \operatorname{tr}\left((\mathbf{X}-\mathbf{M})^{\prime} \boldsymbol{\Sigma}^{-1}(\mathbf{X}-\mathbf{M}) \boldsymbol{\Phi}^{-1}\right)\right) d G(z),
$$

where $h(\cdot)$ is defined by (9) and where $d G(z)=\lambda \exp (-\lambda z) d z, \lambda>0, z \in(0, \infty)$. Then the density generator $h_{1}(\cdot)$ of $\mathbf{X}$ is given by

$$
h_{1}(u)=\frac{\lambda^{\frac{n p}{4}} \Gamma\left(\frac{n p+1}{2}\right)}{2 \pi^{\frac{n p}{2}}} u^{-\frac{n p}{4}} e^{\frac{\lambda u}{2}} \cdot W_{\alpha, \beta}(\lambda u), u \geq 0,
$$

where $W_{\alpha, \beta}(\cdot)$ denotes Whittaker's function with index $\alpha=-\frac{n p+2}{4}$ and $\beta=$ $-\frac{n p-2}{4}$.

Proof. From equation (8) in Gradshteyn and Ryzhik (1965), p. 319, we can write

$$
\begin{aligned}
& h_{1}(u)=\lambda \frac{\Gamma\left(\frac{n p+1}{2}\right)}{\pi^{\frac{n p+1}{2}}} \cdot \lambda^{\frac{n p-4}{4}} \Gamma\left(\frac{3}{2}\right) u^{-\frac{n p}{4}} e^{\frac{\lambda u}{2}} W_{\alpha, \beta}(\lambda u) \\
& =\frac{\lambda^{\frac{n p}{4}} \Gamma\left(\frac{n p+1}{2}\right)}{\pi^{\frac{n p+1}{2}}} \frac{\pi^{\frac{1}{2}}}{2} \cdot u^{-\frac{n p}{4}} e^{\frac{\lambda u}{2}} W_{\alpha, \beta}(\lambda u) \\
& =\frac{\lambda^{\frac{n p}{4}} \Gamma\left(\frac{n p+1}{2}\right)}{2 \pi^{\frac{n p}{2}}} \cdot u^{-\frac{n p}{4}} e^{\frac{\lambda u}{2}} W_{\alpha, \beta}(\lambda u) .
\end{aligned}
$$

The desired result is then obtained.

\section{Some Properties of The SMC Distributions}

Some properties such as stochastic representation and characteristic function are discussed in this section. However, by virtue of relation (2), we shall focus this discussion on the multivariate case only; i.e. when $n=1$. Also, we adopt the notation $\mathbf{x} \sim S M C_{p}\left(\boldsymbol{\mu}, \boldsymbol{\Sigma}, h_{1}\right)$, which means that $\mathbf{x}$ has scale mixture of Cauchy (SMC) distribution with density generator $h_{1}(\cdot)$ given in (13).

3.1. Representation: Distribution of $R^{2}$. Let $\mathbf{x} \sim S M C_{p}\left(\boldsymbol{\mu}, \boldsymbol{\Sigma}, h_{1}\right)$, with $\boldsymbol{\Sigma}>$ O. As a member of the elliptical family, $\mathbf{x}$ admits the stochastic representation

$$
\mathbf{x} \stackrel{d}{=} \boldsymbol{\mu}+R \boldsymbol{\Sigma}^{\frac{1}{2}} \mathbf{u}^{(p)}
$$

where $\boldsymbol{\Sigma}^{\frac{1}{2}}$ is the square root of $\boldsymbol{\Sigma}$. The density of $R^{2}$ is derived in the next lemma.

Lemma 3.1. Let $\mathbf{x} \sim S M C_{p}\left(\boldsymbol{\mu}, \boldsymbol{\Sigma}, h_{1}\right)$. Then the density of $U=R^{2}$ is given by

$$
g(u)=k_{1}(p, \lambda) u^{\frac{p}{4}-1} e^{\frac{\lambda u}{2}} \cdot W_{\alpha, \beta}(\lambda u), u \geq 0,
$$


where $k_{1}(p, \lambda)=\frac{\lambda^{\frac{p}{4}} \Gamma\left(\frac{p+1}{2}\right)}{2 \Gamma\left(\frac{p}{2}\right)}, \alpha=-\frac{p+2}{4}$, and $\beta=-\frac{p-2}{4}$.

Proof. The result is directly obtained from Fang et al. (1990), p. 76, by writing $g(u)=\frac{\pi^{\frac{p}{2}}}{\Gamma\left(\frac{p}{2}\right)} u^{\frac{p}{2}-1} h_{1}(u)$, where $h_{1}(u)$ is given in (13).

Remark 3.1. Using the stochastic representation (14), one can generate a random vector from SMC distributions. Indeed, the density of $R^{2}$ being explicitly given, the uniform distributions can be generated with the help of an efficient algorithm which has been presented in Fang and Wang (1994).

3.2. Moments of $R^{2}$. The following lemma provides the expressions of $E\left(R^{2 s}\right)$.

Lemma 3.2. For any $s>0$, we have

$$
E\left(R^{2 s}\right)=\frac{\Gamma(s+1) \Gamma\left(\frac{p+2 s}{2}\right)}{\sqrt{\pi} \lambda^{s}} .
$$

Proof. From the density of $U=R^{2}$ given in (15), we have

$$
E\left(R^{2 s}\right)=E\left(U^{s}\right)=k_{1}(p, \lambda) \int_{0}^{\infty} u^{\frac{p}{4}+s-1} e^{\frac{\lambda u}{2}} \cdot W_{\alpha, \beta}(\lambda u) d u,
$$

which becomes (after letting $y=\lambda u$ )

$$
E\left(R^{2 s}\right)=\frac{\Gamma\left(\frac{p+1}{2}\right)}{2 \lambda^{s} \Gamma\left(\frac{p}{2}\right)} \int_{0}^{\infty} y^{\frac{p}{4}+s-1} e^{\frac{y}{2}} \cdot W_{\alpha, \beta}(y) d y .
$$

Now, using the following equation (see Gradshteyn and Ryzhik 1965, p. 861, eq. 12),

$$
\int_{0}^{\infty} y^{\nu-1} e^{\frac{y}{2}} \cdot W_{\alpha, \beta}(y) d y=\Gamma(-\alpha-\beta) \frac{\Gamma\left(\frac{1}{2}+\beta+\nu\right) \Gamma\left(\frac{1}{2}-\beta+\nu\right)}{\Gamma\left(\frac{1}{2}-\beta-\alpha\right) \Gamma\left(\frac{1}{2}+\beta-\alpha\right)},
$$

we arrive at the desired result by substituting $\alpha=-\frac{p+2}{4}, \beta=-\frac{p-2}{4}$ and $\nu=\frac{p+4 s}{4}$ in formula (17).

Remark 3.2. Let $R_{c}$ be the generating variate of the $p$-dimensional Cauchy distribution. It has been shown in Fang et al. (1990), p. 82, that $U=R_{c}^{2}$ has a beta type II distribution with parameters $\frac{p}{2}$ and $\frac{1}{2}$. That is, its density function is given by

$$
g(u)=\frac{1}{B\left(\frac{p}{2}, \frac{1}{2}\right)} u^{\frac{p}{2}-1}(1+u)^{-\frac{p+1}{2}}, u>0 .
$$

Hence, the $k$ th moment (if it exists) of $U=R_{c}^{2}$ is evaluated as follows:

$$
E\left(R_{c}^{2 k}\right)=\frac{1}{B\left(\frac{p}{2}, \frac{1}{2}\right)} \int_{0}^{\infty} u^{\frac{p}{2}+k-1}(1+u)^{-\frac{p+1}{2}} d u,
$$

and it is straightforward to see that $E\left(R_{c}^{2 k}\right)=\frac{B\left(\frac{p}{2}+k, \frac{1}{2}-k\right)}{B\left(\frac{p}{2}, \frac{1}{2}\right)}$. Since $k=1,2, \ldots$ and the arguments of beta function $B(\cdot, \cdot)$ must be positive, the $k$ th moment of $R_{c}^{2}$ does not exist.

The first two moments and the multidimensional kurtosis coefficient $\gamma$ of $\mathbf{x}$ are given in the following corollary. 
Corollary 3.1. Let $\mathbf{x} \sim S M C_{p}\left(\boldsymbol{\mu}, \boldsymbol{\Sigma}, h_{1}\right)$. Then we have

$$
E(\mathbf{x})=\boldsymbol{\mu}, \operatorname{Cov}(\mathbf{x})=\boldsymbol{\Sigma}_{1}=\frac{\Gamma\left(\frac{p}{2}\right)}{2 \lambda \sqrt{\pi}} \boldsymbol{\Sigma}, \gamma=\frac{2 p(p+2) \sqrt{\pi}}{\Gamma\left(\frac{p}{2}\right)} .
$$

Proof. The first equality is obvious, while the second arises from the general formula (see Fang et al. (1990), p. 43) $\operatorname{Cov}(\mathbf{x})=\frac{E\left(R^{2}\right)}{\operatorname{rank}(\boldsymbol{\Sigma})} \boldsymbol{\Sigma}$. Here, $\operatorname{rank}(\boldsymbol{\Sigma})=p$, and $E\left(R^{2}\right)$ is given in (16).

The multidimensional kurtosis coefficient $\gamma$ is defined (see Mardia et al. (1979), p. 31) by

$$
\begin{aligned}
\gamma & =E\left(\left[(\mathbf{x}-\boldsymbol{\mu})^{\prime} \boldsymbol{\Sigma}_{1}^{-1}(\mathbf{x}-\boldsymbol{\mu})\right]^{2}\right) \\
& =\frac{p^{2}}{E^{2}\left(R^{2}\right)} \cdot E\left(\left[(\mathbf{x}-\boldsymbol{\mu})^{\prime} \boldsymbol{\Sigma}^{-1}(\mathbf{x}-\boldsymbol{\mu})\right]^{2}\right) .
\end{aligned}
$$

Since $(\mathbf{x}-\boldsymbol{\mu})^{\prime} \boldsymbol{\Sigma}^{-1}(\mathbf{x}-\boldsymbol{\mu}) \stackrel{d}{=} R^{2}, \gamma$ can be written as $\gamma=p^{2} \frac{E\left(R^{4}\right)}{E^{2}\left(R^{2}\right)}$, and hence, from formula (16), we get the desired result.

Now, a result due to Schoenberg (1938), will be used in order to determine the characteristic function of $\mathbf{x} \sim S M C_{p}\left(\boldsymbol{\mu}, \boldsymbol{\Sigma}, h_{1}\right)$.

3.3. Characteristic function of $S M C_{p}\left(\boldsymbol{\mu}, \boldsymbol{\Sigma}, h_{1}\right)$. The characteristic function $\phi_{\mathbf{x}}(\cdot)$ of $\mathbf{x} \sim S M C_{p}(\boldsymbol{\mu}, \boldsymbol{\Sigma})$ has the form (see Fang et al. (1990), p. 32)

$$
\phi_{\mathbf{x}}(\mathbf{t})=\exp \left(i \mathbf{t}^{\prime} \boldsymbol{\mu}\right) \cdot \psi\left(\mathbf{t}^{\prime} \boldsymbol{\Sigma} \mathbf{t}\right)
$$

where $\psi(\cdot)$ denotes the characteristic generator (c.g.) of $\mathbf{x}$. Without loss of generality, here we treat the special case $\boldsymbol{\mu}=\mathbf{0}$ and $\boldsymbol{\Sigma}=\mathbf{I}_{p}$, since $\psi$ does not depend on the choice of $\boldsymbol{\mu}$ and $\boldsymbol{\Sigma}$. A theorem of Schoenberg (1938) and the expression of the c.g. $\Omega_{p}(\cdot)$ of $\mathbf{u}^{(p)}$ given in Fang et al., p. 70, imply in general that

$$
\begin{aligned}
\psi\left(\mathbf{t}^{\prime} \mathbf{t}\right) & =\int_{0}^{\infty} \Omega_{p}\left(\mathbf{t}^{\prime} \mathbf{t} R^{2}\right) d F(R) \\
& =\frac{\Gamma\left(\frac{p}{2}\right)}{\sqrt{\pi}} \sum_{k=0}^{\infty} \frac{(-1)^{k}\left(\mathbf{t}^{\prime} \mathbf{t}\right)^{k}}{(2 k) !} \frac{\Gamma\left(\frac{2 k+1}{2}\right)}{\Gamma\left(\frac{p+2 k}{2}\right)} \cdot \int_{0}^{\infty} R^{2 k} d F(R),
\end{aligned}
$$

where $F(\cdot)$ is the distribution function of $R$, provided that $E\left(R^{2 k}\right)<\infty$ for $k=$ $1,2, \ldots$, and the series on the right hand side is convergent. Now, for the particular case of the scale mixture of Cauchy distribution, we have (see (16))

$$
E\left(R^{2 k}\right)=\frac{k ! \Gamma\left(\frac{p+2 k}{2}\right)}{\sqrt{\pi} \lambda^{k}} .
$$


Substituting (21) in (20) and using the relation $\frac{\Gamma\left(k+\frac{1}{2}\right)}{\sqrt{\pi}(2 k) !}=\frac{1}{4^{k} k !}$, we find

$$
\begin{aligned}
\psi\left(\mathbf{t}^{\prime} \mathbf{t}\right) & =\frac{\Gamma\left(\frac{p}{2}\right)}{\sqrt{\pi}} \sum_{k=0}^{\infty} \frac{(-1)^{k}\left(\mathbf{t}^{\prime} \mathbf{t}\right)^{k}}{(2 k) !} \frac{\Gamma\left(\frac{2 k+1}{2}\right)}{\Gamma\left(\frac{p+2 k}{2}\right)} \cdot \frac{k ! \Gamma\left(\frac{p+2 k}{2}\right)}{\sqrt{\pi} \lambda^{k}} \\
& =\frac{\Gamma\left(\frac{p}{2}\right)}{\sqrt{\pi}} \sum_{k=0}^{\infty}(-1)^{k}\left(\mathbf{t}^{\prime} \mathbf{t}\right)^{k} \frac{\Gamma\left(k+\frac{1}{2}\right)}{\sqrt{\pi}(2 k) !} \cdot \frac{k !}{\lambda^{k}} \\
& =\frac{\Gamma\left(\frac{p}{2}\right)}{\sqrt{\pi}} \sum_{k=0}^{\infty}(-1)^{k}\left(\mathbf{t}^{\prime} \mathbf{t}\right)^{k} \frac{1}{4^{k} k !} \cdot \frac{k !}{\lambda^{k}} \\
& =\frac{\Gamma\left(\frac{p}{2}\right)}{\sqrt{\pi}} \sum_{k=0}^{\infty}\left(-\frac{\mathbf{t}^{\prime} \mathbf{t}}{4 \lambda}\right)^{k} .
\end{aligned}
$$

Hence, we derive a closed form for the characteristic function of $\mathbf{x}$ in the next theorem.

Theorem 3.1. Let $\mathbf{x} \sim S M C_{p}\left(\boldsymbol{\mu}, \boldsymbol{\Sigma}, h_{1}\right)$, with parameters $\boldsymbol{\mu}, \boldsymbol{\Sigma}>\mathbf{O}$, and $\lambda>0$. Then the c.f. of $\mathbf{x}$ is given by

$$
\phi_{\mathbf{x}}(\mathbf{t})=\frac{\Gamma\left(\frac{p}{2}\right)}{\sqrt{\pi}} e^{i \mathbf{t}^{\prime} \boldsymbol{\mu}} \frac{4 \lambda}{4 \lambda+\mathbf{t}^{\prime} \mathbf{\Sigma} \mathbf{t}}, \mathbf{t} \in \mathbb{R}^{p}
$$

provided that $0 \leq \mathbf{t}^{\prime} \boldsymbol{\Sigma} \mathbf{t}<4 \lambda$.

Proof. It suffices to prove that the power series on the right hand side converges under the condition $0 \leq \mathbf{t}^{\prime} \mathbf{\Sigma} \mathbf{t}<4 \lambda$. To this end, consider $b_{k}=\left(-\frac{\mathbf{t}^{\prime} \mathbf{t}}{4 \lambda}\right)^{k}$. Hence, we have

$$
\lim _{k \rightarrow \infty}\left|\frac{b_{k+1}}{b_{k}}\right|=\frac{\mathbf{t}^{\prime} \mathbf{t}}{4 \lambda}=q .
$$

Now, according to the D'Alembert rule (see Gradshteyn and Ryzhik (1965), p. 5), if $q<1$, the series (22) converges. Here $q<1$ is equivalent to $0 \leq \mathbf{t}^{\prime} \boldsymbol{\Sigma} \mathbf{t}<4 \lambda$. The theorem is therefore proved.

\section{Unbiased estimators of $\boldsymbol{\mu}, \boldsymbol{\Sigma}$ And $|\boldsymbol{\Sigma}|$}

We define the $n$-dimensional vector $\mathbf{e}_{n}$ and the $n \times n$ matrix $\mathbf{D}$ as $\mathbf{e}_{n}=(1, \ldots, 1)^{\prime}$, and $\mathbf{D}=\mathbf{I}_{n}-\frac{1}{n} \mathbf{e}_{n} \mathbf{e}_{n}^{\prime}$, where $\mathbf{I}_{n}$ denotes the identity matrix.

Let $\mathbf{x}_{1}, \mathbf{x}_{2}, \ldots, \mathbf{x}_{n}$ be $p$-dimensional random vectors, such that $n>p$ and $\mathbf{x}_{i} \sim$ $S M C_{p}\left(\boldsymbol{\mu}, \mathbf{\Sigma}, h_{1}\right), i=1, \ldots, n$. Moreover, assume that $\mathbf{x}_{i}, i=1, \ldots, n$, are uncorrelated (but not necessarily independent) and that their joint distribution is absolutely continuous. Then, as shown in Gupta and Varga (1993), p. 259, this model can be expressed as

$$
\mathbf{X} \sim S M C_{p, n}\left(\boldsymbol{\mu} \mathbf{e}_{n}^{\prime}, \boldsymbol{\Sigma} \otimes \mathbf{I}_{n}, h_{1}\right),
$$

where $\mathbf{X}=\left(\mathbf{x}_{1}, \mathbf{x}_{2}, \ldots, \mathbf{x}_{n}\right)$ and $h_{1}$ is defined in (13). Now we are interested in the unbiased estimators of the parameters $\boldsymbol{\mu}, \boldsymbol{\Sigma}$, and $|\boldsymbol{\Sigma}|$. To this end, consider the sample mean and the sample sum of products of matrix $\mathbf{S}$ which are defined by $\overline{\mathbf{x}}=\frac{1}{n} \sum_{i=1}^{n} \mathbf{x}_{i}$ and $\mathbf{S}=\sum_{i=1}^{n}\left(\mathbf{x}_{i}-\overline{\mathbf{x}}\right)\left(\mathbf{x}_{i}-\overline{\mathbf{x}}\right)^{\prime}$ respectively. 
For matrix variate elliptical distributions having the structure (23), Anderson and Fang (1982) showed that the unbiased estimators of $\boldsymbol{\mu}$ and $\boldsymbol{\Sigma}$, in general, are given by

$$
\hat{\boldsymbol{\mu}}=\overline{\mathbf{x}}, \widehat{\mathbf{\Sigma}}=\frac{n p}{(n-1) E\left(R^{2}\right)} \mathbf{S},
$$

provided that $0<E\left(R^{2}\right)<\infty$. Also, $\mathbf{X} \stackrel{d}{=} \boldsymbol{\mu} \mathbf{e}_{n}^{\prime}+R \mathbf{A U}$ is the stochastic representation of $\mathbf{X}$. Note that $E\left(R^{2}\right)$ can be obtained from formula (16) by substituting $p n$ for $p$. Hence, $\widehat{\boldsymbol{\Sigma}}$ is given by

$$
\widehat{\mathbf{\Sigma}}=\frac{2 \lambda \sqrt{\pi}}{(n-1) \Gamma\left(\frac{n p}{2}\right)} \mathbf{S} .
$$

The exact distribution of $\hat{\boldsymbol{\mu}}$ and $\mathbf{S}$ are derived in the following theorem.

Theorem 4.1. Let $\mathbf{X} \sim S M C_{p, n}\left(\boldsymbol{\mu} \mathbf{e}_{n}^{\prime}, \boldsymbol{\Sigma} \otimes \mathbf{I}_{n}, h_{1}\right)$, where $h_{1}$ is defined in (13).

(1) Then the joint density of $\hat{\boldsymbol{\mu}}$ and $\mathbf{S}$ is

$$
p(\hat{\boldsymbol{\mu}}, \mathbf{S})=\frac{n^{\frac{p}{2}}|\mathbf{S}|^{\frac{n-p}{2}} \pi^{\frac{p(n-1)}{2}}}{\Gamma_{p}\left(\frac{n-1}{2}\right)|\boldsymbol{\Sigma}|^{\frac{n}{2}}} h_{1}\left(n(\hat{\boldsymbol{\mu}}-\boldsymbol{\mu})^{\prime} \boldsymbol{\Sigma}^{-1}(\hat{\boldsymbol{\mu}}-\boldsymbol{\mu})+\operatorname{tr}\left(\boldsymbol{\Sigma}^{-1} \mathbf{S}\right)\right) .
$$

(2) The p.d.f. of $\hat{\boldsymbol{\mu}}$ is

$$
p_{1}(\hat{\boldsymbol{\mu}})=\frac{\lambda^{\frac{n p}{2}} n^{\frac{p}{2}} v^{\frac{p(n-1)}{2}}}{\pi|\boldsymbol{\Sigma}|^{\frac{1}{2}}} \mathbf{G}_{23}^{31}\left(\lambda v \mid \begin{array}{ccc}
\frac{1-n p}{2}, & 0 \\
-\frac{p(n-1}{2}, & \frac{2-n p}{2}, & 0
\end{array}\right),
$$

where $v=n(\hat{\boldsymbol{\mu}}-\boldsymbol{\mu})^{\prime} \boldsymbol{\Sigma}^{-1}(\hat{\boldsymbol{\mu}}-\boldsymbol{\mu})$.

(3) The p.d.f. of $\mathbf{S}$ is

$$
\begin{aligned}
f(\mathbf{S}) & =\frac{\lambda^{\frac{n p}{2}}|\mathbf{S}|^{\frac{n-p}{2}-1}\left(\operatorname{tr}\left(\boldsymbol{\Sigma}^{-1} \mathbf{S}\right)\right)^{\frac{p}{2}}}{\sqrt{\pi} \Gamma_{p}\left(\frac{n-1}{2}\right)|\boldsymbol{\Sigma}|^{\frac{n-1}{2}}} \mathbf{G}_{23}^{31}\left(\lambda \operatorname{tr}\left(\boldsymbol{\Sigma}^{-1} \mathbf{S}\right) \mid \begin{array}{lll}
\frac{1-n p}{2}, & 0 \\
-\frac{p}{2}, & \frac{2-n p}{2}, & 0
\end{array}\right), \\
& \text { where } G_{q r}^{t s} \text { denotes Meijer's } \mathbf{G} \text {-function. }
\end{aligned}
$$

Proof. From theorem 7.2.1 in Gupta and Varga (1993), p. 239, (1) is obvious. Now, since the proofs of (2) and (3) are similar, we shall give the details only for the latter. Indeed, the p.d.f. of the sample sum of products matrix $\mathbf{S}$ is (see Gupta and Varga (1993), p. 239)

$$
f(\mathbf{S})=\frac{2 \pi^{\frac{p n}{2}}|\mathbf{S}|^{\frac{n-p}{2}}-1}{\Gamma\left(\frac{p}{2}\right) \Gamma_{p}\left(\frac{n-1}{2}\right)|\mathbf{\Sigma}|^{\frac{n-1}{2}}} \int_{0}^{\infty} x^{p-1} h_{1}\left(x^{2}+\operatorname{tr}\left(\boldsymbol{\Sigma}^{-1} \mathbf{S}\right)\right) d x,
$$

where $h_{1}$ is given in (13). Let $b=\operatorname{tr}\left(\boldsymbol{\Sigma}^{-1} \mathbf{S}\right)$, and let $C_{1}=\frac{\lambda^{\frac{n p}{4}} \Gamma\left(\frac{n p+1}{2}\right)}{2 \pi^{\frac{n p}{2}}}$ be the normalizing constant of $h_{1}(\cdot)$. Then, the integral in (27) becomes

$$
\begin{aligned}
\int_{0}^{\infty} & x^{p-1} h_{1}\left(x^{2}+b\right) d x \\
& =C_{1} \int_{0}^{\infty} x^{p-1}\left(x^{2}+b\right)^{-\frac{n p}{4}} \exp \left(\frac{\lambda\left(x^{2}+b\right)}{2}\right) W_{\alpha, \beta}\left(\lambda x^{2}+\lambda b\right) d x \\
& =C_{1} \lambda^{\frac{n p}{4}} e^{\frac{\lambda b}{2}} \int_{0}^{\infty} x^{p-1}\left(\lambda x^{2}+\lambda b\right)^{-\frac{n p}{4}} e^{\frac{\lambda x^{2}}{2}} W_{\alpha, \beta}\left(\lambda x^{2}+\lambda b\right) d x
\end{aligned}
$$


which reduces to (by making a change of variable $y=\lambda x^{2}$ and putting $a=\lambda b$ )

$$
\frac{\lambda^{\frac{n p-2 p}{4}} C_{1} e^{\frac{a}{2}}}{2} \int_{0}^{\infty} y^{\frac{p}{2}-1}(y+a)^{-\frac{n p}{4}} e^{\frac{y}{2}} W_{\alpha, \beta}(y+a) d y .
$$

Now, from equation (6), p. 866 in Gradshteyn and Ryzhik (1965), we can write

$$
\begin{aligned}
\int_{0}^{\infty} y^{\frac{p}{2}-1}(y+a)^{-\frac{n p}{4}} e^{\frac{y}{2}} W_{\alpha, \beta}(y+a) d y \\
\quad=\frac{\Gamma(p / 2) a^{\frac{p}{2}} e^{-\frac{a}{2}}}{\Gamma(3 / 2) \Gamma\left(\frac{n p+1}{2}\right)} \mathbf{G}_{23}^{31}\left(\lambda \operatorname{tr}\left(\boldsymbol{\Sigma}^{-1} \mathbf{S}\right) \mid \begin{array}{ccc}
\frac{1-n p}{2}, & 0 & 0 \\
-\frac{p}{2}, & \frac{2-n p}{2}, & 0
\end{array}\right) .
\end{aligned}
$$

A simple calculation shows that

$$
\begin{aligned}
\frac{\lambda^{\frac{n p-2 p}{4}} C_{1} e^{\frac{a}{2}}}{2} \cdot \frac{\Gamma(p / 2) a^{\frac{p}{2}} e^{-\frac{a}{2}}}{\Gamma(3 / 2) \Gamma\left(\frac{n p+1}{2}\right)} & =\frac{\lambda^{\frac{n p-p}{2}} \Gamma(p / 2) a^{\frac{p}{2}}}{2 \pi^{\frac{n p+1}{2}}} \\
& =\frac{\lambda^{\frac{n p}{2}} \Gamma(p / 2)\left(\operatorname{tr}\left(\boldsymbol{\Sigma}^{-1} \mathbf{S}\right)\right)^{\frac{p}{2}}}{2 \pi^{\frac{n p+1}{2}}} .
\end{aligned}
$$

This implies

$$
\begin{aligned}
\int_{0}^{\infty} x^{p-1} h_{1}\left(x^{2}+b\right) d x \\
\quad=\frac{\lambda^{\frac{n p}{2}} \Gamma(p / 2)\left(\operatorname{tr}\left(\boldsymbol{\Sigma}^{-1} \mathbf{S}\right)\right)^{\frac{p}{2}}}{2 \pi^{\frac{n p+1}{2}}} \mathbf{G}_{23}^{31}\left(\lambda \operatorname{tr}\left(\boldsymbol{\Sigma}^{-1} \mathbf{S}\right) \mid \begin{array}{ccc}
\frac{1-n p}{2}, & 0 \\
-\frac{p}{2}, & \frac{2-n p}{2}, & 0
\end{array}\right) .
\end{aligned}
$$

Consequently, the rest of the proof follows directly by substituting (28) in (27).

The next lemma provides an unbiased estimator of $|\boldsymbol{\Sigma}|$ and an identity involving the special function $\mathbf{G}_{q r}^{t s}$, with matrix argument.

Lemma 4.1. For any $m>0$, the mth moment of $|\mathbf{S}|$ is

(i)

$$
E\left(|\mathbf{S}|^{m}\right)=\frac{2^{m(p-1)} m ! \Gamma\left(\frac{n p}{2}\right) \Gamma_{p}\left(\frac{n-1}{2}+m\right)}{\sqrt{\pi} \lambda^{m} \Gamma_{p}\left(\frac{n-1}{2}\right)}|\boldsymbol{\Sigma}|^{m} .
$$

(ii) The unbiased estimator of $\delta=|\boldsymbol{\Sigma}|$ is

$$
\hat{\delta}=\frac{2 \lambda \sqrt{\pi}}{\Gamma\left(\frac{n p}{2}\right)} \frac{\Gamma(n-p)}{\Gamma(n)}|\mathbf{S}| .
$$

(iii)

$$
\begin{aligned}
& \int_{\mathbf{S}>\mathbf{O}}|\mathbf{S}|^{\frac{2 m+n-p}{2}-1}\left(\operatorname{tr}\left(\boldsymbol{\Sigma}^{-1} \mathbf{S}\right)^{\frac{p}{2}} \mathbf{G}_{23}^{31}\left(\lambda \operatorname{tr}\left(\boldsymbol{\Sigma}^{-1} \mathbf{S}\right) \mid \begin{array}{ccc}
\frac{1-n p}{2}, & 0 \\
-\frac{p}{2}, & \frac{2-n p}{2}, & 0
\end{array}\right) d \mathbf{S}\right. \\
& =\frac{2^{m(p-1)} m ! \Gamma\left(\frac{n p}{2}\right) \Gamma_{p}\left(\frac{n-1}{2}+m\right)}{\lambda^{\frac{n p+2 m}{2}}}|\boldsymbol{\Sigma}|^{\frac{2 m+n-1}{2}} .
\end{aligned}
$$

Proof. Before going into the details, recall that $\mathbf{S}$ can be expressed as $\mathbf{S}=\mathbf{X D X}^{\prime}$, where $\mathbf{D}$ is defined in the beginning of this section. To prove (29), consider the 
function $K(\cdot)$ defined as $K(\mathbf{X})=\left|\mathbf{X D X}^{\prime}\right|^{m}$. Thus, for $a>0$, we have $K(a \mathbf{X})=$ $a^{2 m} K(\mathbf{X})$. Using the result presented in Gupta and Varga (1993), p. 100, we can write

$$
E(K(\mathbf{X}))=\frac{E\left(R^{2 m}\right) \Gamma\left(\frac{n p}{2}\right)}{2^{m} \Gamma\left(\frac{n p+2 m}{2}\right)} E(K(\mathbf{Y})),
$$

where $K(\mathbf{Y}) \stackrel{d}{=}\left|\mathbf{W}_{p}(n-1, \boldsymbol{\Sigma})\right|^{m}$. Here, the symbol $\mathbf{W}_{p}$ stands for the Wishart matrix. Since $E(K(\mathbf{Y}))$ is given (see Gupta and Nagar (2000), p. 105) by

$$
E(K(\mathbf{Y}))=\frac{2^{p m} \Gamma_{p}\left(\frac{n-1}{2}+m\right)}{\Gamma_{p}\left(\frac{n-1}{2}\right)}|\boldsymbol{\Sigma}|^{m}
$$

and

$$
E\left(R^{2 m}\right)=\frac{m ! \Gamma\left(\frac{n p+2 m}{2}\right)}{\sqrt{\pi} \lambda^{m}},
$$

then (29) is obtained by substituting (34) and (33) in (32). The special case where $m=1$ in (29) leads to the unbiased estimator $\hat{\delta}$. Finally, (31) can be easily obtained by writing

$$
E\left(|\mathbf{S}|^{m}\right)=\int_{\mathbf{S}>\mathbf{O}}|\mathbf{S}|^{m} f(\mathbf{S}) d \mathbf{S} .
$$

The lemma is then proved.

\section{Concluding Remarks}

In this paper, we have introduced and investigated a new subclass of matrix variate elliptical distributions. Such a subclass is obtained by making use of a mixture method. In spite of the presence of a special function in the expression of the density generator $h_{1}(\cdot)$, namely Whittaker's function, simple closed forms have been derived both for the characteristic function and for the moments of the generating variate $R$. Unlike the Cauchy distribution, which suffers from limited applicability because of the lack of finite moments (see Remark 3.2), the SMC distribution could therefore be a better model for certain practical situations.

If, in formula (22), $p=1, \boldsymbol{\Sigma}=\sigma^{2}, \boldsymbol{\mu}=0$, and $\lambda=\frac{1}{2}$, then the c.f. $\phi_{x}(t)=$ $\frac{1}{1+\frac{\sigma^{2} t^{2}}{2}}$ is the characteristic function of a univariate Laplace distribution with mean zero and variance $\sigma^{2}$ (see Kotz et al. (2003)). Moreover, when $p=1$ in (18), then $\gamma$ equals 6 , which is also the kurtosis coefficient of the univariate Laplace distribution (see Krishnamoorthy (2006)). Hence, because of these remarks, the scale mixture of Cauchy distribution can be viewed as an extension of the univariate Laplace distribution.

Further details regarding the special functions that have been used in this work can be found in Abramowitz and Stegun (1965) or in Gradshteyn and Ryzhik (1965).

\section{ACKNOWLEDGMENTS}

The authors would like to thank the anonymous referee and the editor for their very valuable comments and suggestions, which improved the presentation of the paper. 


\section{REFERENCES}

[1] Abramowitz, M. and Stegun, I.A. (1965). Handbook of Mathematical Functions, with Formulas, Graphs and Mathematical Tables. Dover Publications, New York. MR0167642 (29:4914)

[2] Anderson, T.W. and Fang, K.T. (1982). On the Theory of Multivariate Elliptically Contoured Distributions and Their Applications. Technical Report 54. Department of Statistics, Stanford University, California. Reprinted in Fang and Anderson (1990). MR.1066888 (92f:62068)

[3] Fang, K.T. and Anderson, T.W. (1990). Statistical Inference in Elliptically Contoured and Related Distributions. Allerton Press, New York. MR1066887 (91e:62006)

[4] Fang, K.T., Kotz, S. and Ng, K.W. (1990). Symmetric Multivariate and Related Distributions. Chapman and Hall, London, New York. MR 1071174 (91i:62070)

[5] Fang, K.T. and Zhang, Y.T. (1990). Generalized Multivariate Analysis. Springer-Verlag, New York. MR 1079542 (92f:62066)

[6] Fang, K.T. and Wang, Y. (1994). Number-Theoretic Methods in Statistics. Chapman and Hall, London. MR1284470 (95g:65189)

[7] Gradshteyn, I.S. and Ryzhik, I.M. (1965). Tables of Integrals, Series, and Products. Academic Press, New York. MR0197789 (33:5952)

[8] Gupta, A.K. and Nagar, D.K. (2000). Matrix Variate Distributions. Chapman and Hall, Boca Raton, FL. MR:1738933 (2001d:62055)

[9] Gupta, A.K. and Varga, T. (1993). Elliptically Contoured Models in Statistics. Kluwer Academic Publishers, Dordrecht. MR1256485 (95a:62038)

[10] Gupta, A.K. and Varga, T. (1995). Normal Mixture of Matrix Variate Elliptically Contoured Distributions. Sankhya, Ser. A 57, 68-78. MR1392632 (97f:62095)

[11] Kotz, S., Kozubowski, T.J. and Podgorski, K. (2003). An Asymmetric Multivariate Laplace Distribution. Technical Report 367 (January 2003). http://wolfweb. unr.edu/homepage/tkozubow/o_alm.pdf

[12] Krishnamoorthy, K. (2006). Handbook of Statistical Distributions with Applications. Chapman and Hall/CRC. MR2259696 (2007c:62001)

[13] Mardia, K.V., Kent, J.T. and Bibby, J.M. (1979). Multivariate Analysis, Academic Press, London. MR.560319 (81h:62003)

[14] Muirhead, R.J. (1982). Aspects of Multivariate Statistical Theory. Wiley, New York. MR652932 (84c:62073)

[15] Schoenberg, I.J. (1938). Metric Spaces and Completely Monotone Functions. Ann. Math. 39, 811-841. MR.1503439

[16] Streit, F. (1991). On the Characteristic Functions of the Kotz-type Distributions. C.R. Math. Rep. Acad. Sci. Canada 13, 121-124. MR.1124723(93c:60019)

Department of Mathematics and Statistics, McMaster University, Hamilton, OnTARIO L8S 4K1, CANADA

E-mail address: asarr@math.mcmaster.ca

Department of Mathematics and Statistics, Bowling Green State University, BowlING Green, Ohio 43403

E-mail address: gupta@bgsu.edu 